\title{
Hydrothermal Synthesis, Characterization and Photoluminescent Properties of a New Vanadium Substituted Keggin Assembly Supported by Ni Complex
}

\author{
Imen Soussi ${ }^{\mathrm{a}^{*}}$, Sameh Aoun ${ }^{\mathrm{b}}$, Aurelien Planchat ${ }^{\mathrm{b}}$ and Samah Akriche ${ }^{\mathrm{a}}$ \\ ${ }^{a}$ Laboratoire de Chimie des Matériaux, Département Chimie, Faculté des Sciences de Bizerte, 7021 \\ Zarzouna, Tunisie \\ ${ }^{\mathrm{b}}$ Laboratoire CEISAM, UMR CNRS 6230, UFR des sciences et des techniques, 2 rue de la Houssinière BP \\ 9220844322 Nantes cedex 03
}

\section{ABSTRACT}

A new monosubstituted vanadium Keggin-type tungstophosphate derivative, $\left[\mathrm{Ni}(\text { phen })_{3}\right]_{2}\left[\mathrm{PVW}_{11} \mathrm{O}_{40}\right] \cdot 4 \mathrm{H}_{2} \mathrm{O}$ (phen $=1,10^{\prime}-$ phenanthroline), has been hydrothermally synthesized and characterized by powder and single-crystal XRD methods, scanning electron microscopy (SEM), IR, UV-Vis and photoluminescence spectroscopy measurements. X-ray diffraction analysis reveals that the compound crystallizes in the monoclinic system with space group C2/c with $a=19.659$ (2) $\AA$, $b=$ 18.054 (3) $\AA, c=25.227$ (4) $\AA$ and $\beta=100.63(2)^{\circ}$ and its crystal packing displays alternating $\left[\mathrm{PV}_{1} \mathrm{~W}_{11} \mathrm{O}_{40}\left(\mathrm{H}_{2} \mathrm{O}\right)_{4}\right]_{n}{ }^{4}$ ribbons extending along [110] and [110] respectively at $z=0$ and $z=1 / 2$. These ribbons are extended into a 3D supramolecular framework by hydrogen-bonding interactions generating vacant 1D-channels along c-axis leading to anchorage of transition metal complexes. Moreover, the electronic properties and the optical band gaps have also been investigated well confirming the semiconductor behavior and photoluminescent property of reported material.

\section{Indexing terms/Keywords}

Hydrothermal synthesis; Polyoxometalates; Substituted Keggin; Optical band gap, Photoluminescent properties

\section{Academic Discipline And Sub-Disciplines}

Provide examples of relevant academic disciplines for this journal: E.g., History; Education; Sociology; Psychology; Cultural Studies;

\section{SUBJECT CLASSIFICATION}

E.g., Mathematics Subject Classification; Library of Congress Classification

\section{TYPE (METHOD/APPROACH)}

Provide examples of relevant research types, methods, and approaches for this field: E.g., Historical Inquiry; QuasiExperimental; Literary Analysis; Survey/Interview

\section{Council for Innovative Research}

Peer Review Research Publishing System

Journal: Journal of Advances in Chemistry

Vol. 10, No. 1

editorjaconline@gmail.com

www.cirjac.com 


\section{INTRODUCTION}

Polyoxometalates (POMs) have attracted great attention in recent years owing to their potential applications in catalysis, photochemistry, magnetism, electrical conductivity and biological chemistry [1-8]. Polyoxometalates mainly those displaying the Keggin structure, are very interesting and exhibit applications in many fields such as medicinal, acid catalysis and redox catalysis [9-13]. In order to adjust their properties, the keggin clusters are often substituted by some selected transition metals. The resulting substituted Keggin-type $\left[\mathrm{XM}_{\mathrm{n}} \mathrm{W}_{12-\mathrm{n}} \mathrm{O}_{40}\right]^{\mathrm{p}-}$ heteropolyoxotungstate clusters, are shown in a lot of cases, more active than their precursor. In particular, when substituted by vanadium metal, additional properties (antimicrobial, redox, acidity, stabilization, etc.) can be observed in many oxidations, biological and catalytic processes [14-19]. However, the controls of their formation conditions are rather difficult when they are combined to metalorganic frameworks. A bibliographic survey shows only a few examples of monosubstituted vanadium keggin-type $\left[\mathrm{XV}_{1} \mathrm{~W}_{11} \mathrm{O}_{40}\right]^{\mathrm{p}-}$ clusters associated to organometallic or organic moieties, such as $\left(\mathrm{C}_{2} \mathrm{~N}_{2} \mathrm{H}_{10}\right)_{2}\left[\mathrm{VV}_{1} \mathrm{~W}_{11} \mathrm{O}_{40}\right] .6 \mathrm{H}_{2} \mathrm{O}$ [20], $\left[\mathrm{N}\left(\mathrm{CH}_{3}\right)_{4}\right]_{4}\left[\mathrm{VV}_{1} \mathrm{~W}_{11} \mathrm{O}_{40}\right] .4 .5 \mathrm{H}_{2} \mathrm{O}[21],\left[\left(\mathrm{CH}_{3}\right)_{2} \mathrm{NCHO}_{4} \mathrm{H}_{4}\left[\mathrm{VW}_{11} \mathrm{~V}_{1} \mathrm{O}_{40}\right] .2\left[\left(\mathrm{CH}_{3}\right)_{2} \mathrm{NCHO}\right] .2 \mathrm{H}_{2} \mathrm{O}[22],\left[\mathrm{Cu}(\text { phen })_{2}\right]_{2} \mathrm{PVW}_{11} \mathrm{O}_{40}[23]\right.$ and $\left(\mathrm{n}-\mathrm{Bu}_{4} \mathrm{~N}\right)_{4}\left[\mathrm{PMW}_{11} \mathrm{O}_{40}\right](\mathrm{M}=\mathrm{V}, \mathrm{Nb}, \mathrm{Ta})[24]$.

Intrigued by these results, we attempted to explore our research on the preparation of vanadium-substituted Keggin-type tungstophosphate materials using multidentate $\mathrm{N}$-donor ligands and with the aim of achieving luminescent properties; we selected the well-known bidentate heterocyclic N-donor ligand, the phenanthroline (phen). In the course of these investigations and by varying the metal chelating agent, we succeeds to prepare new two Keggin-type tungstovanado phosphate cluster decorated by metal-organic mononuclear complex. The first with Co ion was recently published $\left.[\mathrm{Co} \text { (phen) }]_{2}\right]_{2}\left[\mathrm{PVW}_{11} \mathrm{O}_{40}\right] \cdot 2 \mathrm{H}_{2} \mathrm{O}[25]$ and the second with $\mathrm{Ni}$ metal; [ $\left.\mathrm{Ni}(\text { phen })_{3}\right]_{2}\left[\mathrm{PVW}_{11} \mathrm{O}_{40}\right] \cdot 4 \mathrm{H}_{2} \mathrm{O}$ is the subject of this paper. Its preparation and its structure and physicochemical characterization are discussed.

\section{EXPERIMENTAL SECTION}

\section{1. Synthesis of $\left[\mathrm{Ni}(\text { phen })_{3}\right]_{2}\left[\mathrm{PVW}_{11} \mathrm{O}_{40}\right] .4 \mathrm{H}_{2} \mathrm{O}$}

All reagents used were purchased and used without further purification. A reaction mixture of $\mathrm{Na}_{2} \mathrm{WO}_{4} .2 \mathrm{H}_{2} \mathrm{O}(2 \mathrm{~g}$ $6 \mathrm{mmol}), \mathrm{NaH}_{2} \mathrm{PO}_{4} .2 \mathrm{H}_{2} \mathrm{O}(0.156 \mathrm{~g}, 1 \mathrm{mmol}), \mathrm{NiCl}_{2} \cdot 6 \mathrm{H}_{2} \mathrm{O}(0.24 \mathrm{~g}, 1 \mathrm{mmol}), \mathrm{V}_{2} \mathrm{O}_{5}(0,045 \mathrm{~g} ; 0.25 \mathrm{mmol})$ and phen. $\mathrm{H}_{2} \mathrm{O}(0.6$ $\mathrm{g}, 3 \mathrm{mmol}$ ) were added to water $(10 \mathrm{~mL})$. The mixture was adjusted to $\mathrm{pH}=5.5$ by the addition of $4 \mathrm{~mol} \mathrm{~L}^{-1} \mathrm{HCl}_{\text {aqueous }}$ solution; then stirred for $30 \mathrm{~min}$ in air. The solution was transferred into a $23 \mathrm{~mL}$ Teflon-lined autoclave and crystallized at $180^{\circ} \mathrm{C}$ for 4 days. Then the autoclave was cooled at $10^{\circ} \mathrm{C} \mathrm{h}^{-1}$ to room temperature. The resulting dark block crystals of 1 were filtered off, washed with water, and dried at ambient temperature to give yields of $68 \%$ based on W. Anal. Calc. For $\mathrm{C}_{72} \mathrm{H}_{56} \mathrm{~N}_{12} \mathrm{Ni}_{2} \mathrm{O}_{44} \mathrm{PVW}_{11}(\%)$ : C, 21.52; H, 1.39; N, 4.18; W, 50.41; V, 1.27; P, 0.77; Ni, 2.94. Found C, 21.76; H, 1.29; N, 4.18; W, 50.20; V, 1.30; P, 0.8; Ni, 2.98.

\section{2. X-Ray crystal structure determination}

A single crystal of the title compound, $\left[\mathrm{Ni}\left(\mathrm{C}_{12} \mathrm{H}_{8} \mathrm{~N}_{2}\right)_{3}\right]_{2}\left[\mathrm{PVW}_{11} \mathrm{O}_{40}\right] \cdot 4 \mathrm{H}_{2} \mathrm{O}$, was glued to fiber glass for data collection at room temperature on a Nonius KappaCCD diffractometer with graphite-monochromated MoKa $(\lambda=071073 \AA)$ radiation at room temperature The structure was solved by Shelxs97 [26] in the Wingx package [27] and refined by fullmatrix least-squares techniques on $\mathrm{F}^{2}$ with the help of the Shelxl97 [27] crystallographic software package All the nonhydrogen atoms were refined anisotropically Hydrogen atoms of organic ligands were located in the calculated positions and refined in a riding mode where the hydrogen atoms of water molecule were located by finding difference Fourier maps. A summary of the crystallographic data and structural determination for 1 is listed in Table 1 . The hydrogen bonding scheme and selected bond lengths and angles are given respectively in Tables 2 and 3 . The molecular graphics are drawn using Diamond [28].

Table 1. Crystal data, data collection and structure refinement details for $\left[\mathrm{Ni}\left(\mathrm{C}_{12} \mathrm{H}_{8} \mathrm{~N}_{2}\right)_{3}\right]_{2}\left[\mathrm{PVW} \mathrm{W}_{11} \mathrm{O}_{40}\right] .4 \mathrm{H}_{2} \mathrm{O}$

\begin{tabular}{ll}
\hline Chemical formula & {$\left[\mathrm{Ni}\left(\mathrm{C}_{12} \mathrm{H}_{8} \mathrm{~N}_{2}\right)_{3}\right]_{2}\left[\mathrm{PVW}{ }_{11} \mathrm{O}_{40}\right] .4 \mathrm{H}_{2} \mathrm{O}$} \\
Formula weight & 4014.97 \\
Crystal size, $\mathrm{mm}^{3}$ & $0.3 \times 0.2 \times 0.1$ \\
Crystal system & Monoclinic \\
Space group & $C 2 / \mathrm{c}$ \\
$a, \AA$ & $19.659(2)$ \\
$b, \AA$ & $18.054(3)$ \\
$C, \AA$ & $25.227(4)$ \\
$\beta,{ }^{\circ}$ & $100.63(2)$ \\
$V, \AA^{3}$ & $8800(2)$ \\
$Z$ & 4 \\
$D_{\text {calcd }} \mathrm{g} \mathrm{cm}^{-3}$ & 3.004
\end{tabular}




$\begin{array}{ll}\mu\left(\mathrm{Mo} K_{\alpha}\right), \mathrm{mm}^{-1} & 14.95 \\ F(000) & 7328 \\ \theta \text { range for data collection, }{ }^{\circ} & 6.4-29.9 \\ \text { Range of } \mathrm{h}, \mathrm{k}, \mathrm{I} & -27 / 27,-24 / 25,-35 / 35 \\ \text { Reflections collected } & 81604 \\ \text { Independent reflections } & 12441 \\ \text { Absorption correction } & \text { multi-scan } \\ \mathrm{T}_{\min / \text { max }} & 0.053 / 0.145 \\ \mathrm{Final} R\left[\mathrm{~F}^{2}>2 \sigma\left(F^{2}\right)\right] / w R\left(F^{2}\right) & 0.099 / 0.214 \\ \mathrm{GoF}\left(\mathrm{F}^{2}\right)^{\mathrm{c}} & 1.19 \\ \Delta \rho_{\max / \min }, \mathrm{e} \AA^{-3} & 2.70 /-2.86\end{array}$

Table 2. Atomic coordinates and $\mathrm{U}_{\text {eq }}$ or $\mathrm{U}_{\text {iso }}$ for $\left[\mathrm{Ni}\left(\mathrm{C}_{12} \mathrm{H}_{8} \mathrm{~N}_{2}\right)_{3}\right]_{2}\left[\mathrm{PVW}_{11} \mathrm{O}_{40}\right] \cdot 4 \mathrm{H}_{2} \mathrm{O}$

\begin{tabular}{|c|c|c|c|c|c|}
\hline & $x$ & $y$ & $z$ & $U_{\text {iso }}{ }^{*} / U_{\text {ea }}$ & Occ. $(<1)$ \\
\hline W1 & $0.83198(4)$ & $0.10244(5)$ & $0.57959(4)$ & $0.0360(2)$ & 0.91 \\
\hline V1 & $0.83198(4)$ & $0.10244(5)$ & $0.57959(4)$ & $0.0360(2)$ & 0.09 \\
\hline W2 & $0.67662(5)$ & $0.17989(6)$ & $0.60800(4)$ & $0.0404(2)$ & 0.91 \\
\hline V2 & $0.67662(5)$ & $0.17989(6)$ & $0.60800(4)$ & $0.0404(2)$ & 0.09 \\
\hline W3 & $0.91117(4)$ & $0.27937(5)$ & $0.58812(3)$ & $0.02974(18)$ & 0.92 \\
\hline V3 & $0.91117(4)$ & $0.27937(5)$ & $0.58812(3)$ & $0.02974(18)$ & 0.08 \\
\hline W4 & $0.90317(4)$ & $0.16944(5)$ & $0.46972(4)$ & $0.03122(19)$ & 0.92 \\
\hline V4 & $0.90317(4)$ & $0.16944(5)$ & $0.46972(4)$ & $0.03122(19)$ & 0.08 \\
\hline W5 & $0.67292(5)$ & $0.07262(5)$ & 0.48950 (5) & $0.0414(2)$ & 0.92 \\
\hline V5 & $0.67292(5)$ & $0.07262(5)$ & $0.48950(5)$ & $0.0414(2)$ & 0.08 \\
\hline W6 & $0.74374(6)$ & $0.14291(6)$ & $0.38101(4)$ & $0.0414(2)$ & 0.92 \\
\hline V6 & $0.74374(6)$ & $0.14291(6)$ & $0.38101(4)$ & $0.0414(2)$ & 0.08 \\
\hline $\mathrm{Ni1}$ & $0.69445(15)$ & 0.18895 (17) & $0.84348(12)$ & $0.0402(6)$ & \\
\hline O1E & $0.8722(11)$ & $0.0303(10)$ & $0.6152(9)$ & $0.065(6)$ & \\
\hline O2E & $0.6446(9)$ & 0.1469 (12) & $0.6596(7)$ & $0.056(5)$ & \\
\hline O3E & $0.9857(9)$ & 0.2933 (13) & $0.6306(8)$ & $0.069(6)$ & \\
\hline O4E & $0.9757(9)$ & $0.1321(10)$ & $0.4554(10)$ & $0.069(6)$ & \\
\hline O5E & $0.6370(8)$ & $-0.0101(9)$ & $0.4866(8)$ & $0.053(5)$ & \\
\hline O6E & $0.7433(10)$ & $0.0950(12)$ & $0.3246(8)$ & $0.065(6)$ & \\
\hline 01 & $0.7636(8)$ & 0.1329 (14) & $0.6191(8)$ & $0.074(7)$ & \\
\hline $\mathrm{O} 2$ & $0.6454(9)$ & 0.1049 (15) & $0.5540(10)$ & $0.088(9)$ & \\
\hline O3A & $0.8468(13)$ & 0.1170 (17) & $0.4101(12)$ & $0.026(6)$ & 0.50 \\
\hline О3В & 0.8224 (17) & 0.1323 (17) & $0.4244(12)$ & $0.032(7)$ & 0.50 \\
\hline O4 & $0.8812(10)$ & $0.1034(9)$ & $0.5227(6)$ & $0.050(4)$ & \\
\hline
\end{tabular}




\begin{tabular}{|c|c|c|c|c|c|}
\hline O5 & 0.9397 (9) & $0.2344(8)$ & $0.5285(6)$ & $0.044(4)$ & \\
\hline O6 & $0.8859(10)$ & $0.1851(10)$ & $0.6093(7)$ & 0.054 & \\
\hline $\mathrm{O} 7$ & $0.7598(9)$ & $0.0536(14)$ & $0.5319(9)$ & $0.076(7)$ & \\
\hline O8 & $0.7128(12)$ & $0.0758(13)$ & $0.4294(11)$ & $0.090(9)$ & \\
\hline O9 & $0.7238(12)$ & $0.2658(12)$ & $0.6395(9)$ & $0.085(8)$ & \\
\hline 010 & $0.6067(11)$ & $0.2449(11)$ & $0.5752(8)$ & $0.074(7)$ & \\
\hline 011 & $0.8475(12)$ & $0.3184(14)$ & $0.6297(10)$ & 0.091 (9) & \\
\hline $\mathrm{O} 12 \mathrm{~A}$ & $0.6008(16)$ & $0.1293(13)$ & $0.4503(10)$ & $0.108(11)$ & \\
\hline P1 & 0.7500 & 0.2500 & 0.5000 & $0.0117(9)$ & \\
\hline O1C & $0.7966(13)$ & $0.3035(17)$ & $0.5399(10)$ & $0.030(6)$ & 0.48 \\
\hline O3C & $0.7403(14)$ & $0.1790(17)$ & $0.5325(11)$ & $0.040(7)$ & 0.52 \\
\hline $\mathrm{O} 2 \mathrm{C}$ & $0.7057(12)$ & $0.2702(13)$ & $0.5442(10)$ & $0.021(5)$ & 0.47 \\
\hline $\mathrm{O} 4 \mathrm{C}$ & $0.8157(11)$ & $0.2158(16)$ & $0.5247(9)$ & $0.031(6)$ & 0.53 \\
\hline O1W & $0.934(2)$ & $-0.0998(18)$ & $0.6138(12)$ & $0.179(18)$ & \\
\hline H1W1 & 0.9096 & -0.0684 & 0.6256 & $0.268^{*}$ & \\
\hline $\mathrm{H} 2 \mathrm{~W} 1$ & 0.9564 & -0.1174 & 0.5919 & $0.268^{*}$ & \\
\hline O2W & $0.6129(13)$ & $0.4552(9)$ & $0.6391(7)$ & $0.174(17)$ & \\
\hline H1W2 & 0.6402 & 0.4208 & 0.6525 & $0.262^{*}$ & \\
\hline H2W2 & 0.5908 & 0.4402 & 0.6088 & $0.262^{*}$ & \\
\hline N1 & 0.7799 (9) & $0.1756(10)$ & $0.8037(7)$ & $0.034(4)$ & \\
\hline N2 & $0.7692(9)$ & $0.1319(11)$ & $0.9032(8)$ & $0.040(4)$ & \\
\hline N3 & $0.6398(10)$ & $0.0894(14)$ & $0.8168(9)$ & $0.054(6)$ & \\
\hline N4 & $0.6210(9)$ & $0.1853(10)$ & $0.8950(8)$ & $0.039(4)$ & \\
\hline N5 & $0.6344(10)$ & $0.2554(12)$ & $0.7822(9)$ & $0.046(5)$ & \\
\hline N6 & $0.7373(11)$ & $0.2945(12)$ & $0.8653(9)$ & $0.048(5)$ & \\
\hline $\mathrm{C} 12$ & $0.8364(10)$ & $0.1441(11)$ & $0.8322(9)$ & $0.036(4)$ & \\
\hline C22 & $0.6138(13)$ & $0.2307(14)$ & $0.9337(11)$ & $0.049(6)$ & \\
\hline $\mathrm{H} 22$ & 0.6412 & 0.2730 & 0.9384 & $0.059^{*}$ & \\
\hline C18 & $0.4925(13)$ & $0.0373(16)$ & $0.9131(14)$ & $0.058(7)$ & \\
\hline $\mathrm{H} 18$ & 0.4618 & 0.0245 & 0.9356 & $0.070^{*}$ & \\
\hline C24 & $0.5913(12)$ & $0.0728(14)$ & $0.8466(9)$ & $0.044(5)$ & \\
\hline C11 & $0.8297(10)$ & $0.1198(17)$ & $0.8878(9)$ & $0.049(7)$ & \\
\hline $\mathrm{C} 2$ & $0.8465(12)$ & $0.195(2)$ & $0.7345(10)$ & $0.065(9)$ & \\
\hline $\mathrm{H} 2$ & 0.8492 & 0.2145 & 0.7008 & $0.078^{*}$ & \\
\hline C20 & $0.5252(11)$ & $0.1544(15)$ & $0.9622(12)$ & $0.051(6)$ & \\
\hline $\mathrm{H} 2 \mathrm{O}$ & 0.4944 & 0.1445 & 0.9853 & $0.061^{*}$ & \\
\hline C19 & $0.5313(12)$ & $0.1064(13)$ & $0.9208(11)$ & $0.047(6)$ & \\
\hline
\end{tabular}




\begin{tabular}{|c|c|c|c|c|}
\hline C35 & $0.7112(12)$ & $0.3465(12)$ & $0.8288(9)$ & $0.038(5)$ \\
\hline C36 & $0.6567(13)$ & $0.3279(19)$ & $0.7841(11)$ & $0.061(8)$ \\
\hline $\mathrm{C} 13$ & $0.6521(13)$ & $0.0420(14)$ & $0.7799(10)$ & $0.048(6)$ \\
\hline $\mathrm{H} 13$ & 0.6862 & 0.0525 & 0.7600 & $0.057^{*}$ \\
\hline C23 & $0.5814(10)$ & $0.1239(12)$ & $0.8891(10)$ & $0.039(5)$ \\
\hline C21 & $0.5666(12)$ & $0.2191(13)$ & 0.9691 (12) & $0.053(7)$ \\
\hline $\mathrm{H} 21$ & 0.5629 & 0.2532 & 0.9961 & $0.064^{*}$ \\
\hline C10 & $0.7648(14)$ & $0.113(2)$ & $0.9537(10)$ & $0.063(8)$ \\
\hline $\mathrm{H} 10$ & 0.7232 & 0.1208 & 0.9654 & $0.075^{*}$ \\
\hline $\mathrm{C7}$ & $0.8869(11)$ & $0.0871(14)$ & $0.9209(10)$ & $0.046(6)$ \\
\hline C1 & $0.7856(13)$ & $0.2014(16)$ & $0.7545(10)$ & $0.051(6)$ \\
\hline $\mathrm{H} 1$ & 0.7475 & 0.2241 & 0.7333 & $0.061^{*}$ \\
\hline C3 & $0.9016(11)$ & $0.1607(16)$ & $0.7634(10)$ & $0.050(6)$ \\
\hline $\mathrm{H} 3$ & 0.9415 & 0.1539 & 0.7490 & $0.059^{*}$ \\
\hline C17 & $0.5021(11)$ & $-0.0075(16)$ & $0.8725(14)$ & $0.062(9)$ \\
\hline $\mathrm{H} 17$ & 0.4751 & -0.0498 & 0.8655 & $0.074^{*}$ \\
\hline C28 & $0.6300(17)$ & $0.3784(19)$ & $0.7474(13)$ & $0.070(9)$ \\
\hline C5 & $0.9538(14)$ & $0.1002(16)$ & $0.8512(14)$ & $0.063(8)$ \\
\hline H5 & 0.9958 & 0.0950 & 0.8396 & $0.075^{\star}$ \\
\hline C16 & $0.5524(12)$ & $0.0076(14)$ & $0.8396(12)$ & $0.049(6)$ \\
\hline C14 & $0.6143(15)$ & $-0.0242(16)$ & $0.7705(12)$ & $0.058(7)$ \\
\hline $\mathrm{H} 14$ & 0.6230 & -0.0561 & 0.7435 & $0.070^{*}$ \\
\hline C25 & $0.5884(13)$ & $0.2368(18)$ & $0.7417(11)$ & $0.055(7)$ \\
\hline $\mathrm{H} 25$ & 0.5749 & 0.1874 & 0.7399 & $0.066^{*}$ \\
\hline C29 & $0.6555(19)$ & $0.4563(16)$ & $0.7523(14)$ & $0.065(9)$ \\
\hline $\mathrm{H} 29$ & 0.6364 & 0.4923 & 0.7277 & $0.078^{*}$ \\
\hline C34 & $0.7896(16)$ & $0.3169(19)$ & $0.9025(12)$ & $0.063(7)$ \\
\hline H34 & 0.8100 & 0.2816 & 0.9272 & $0.076^{*}$ \\
\hline C27 & $0.5767(15)$ & $0.3540(19)$ & $0.7027(13)$ & $0.074(10)$ \\
\hline H27 & 0.5571 & 0.3874 & 0.6762 & $0.089^{*}$ \\
\hline $\mathrm{C} 8$ & $0.8780(18)$ & $0.0708(17)$ & $0.9727(13)$ & $0.072(10)$ \\
\hline $\mathrm{H} 8$ & 0.9147 & 0.0506 & 0.9969 & $0.087^{*}$ \\
\hline C4 & $0.8987(13)$ & $0.1358(15)$ & $0.8140(11)$ & $0.051(6)$ \\
\hline C32 & $0.795(2)$ & $0.4372(16)$ & $0.8771(18)$ & $0.082(12)$ \\
\hline H32 & 0.8135 & 0.4844 & 0.8823 & $0.099^{*}$ \\
\hline C31 & $0.7389(19)$ & $0.4215(13)$ & $0.8324(14)$ & $0.068(9)$ \\
\hline C15 & $0.5646(13)$ & $-0.0431(15)$ & $0.8003(14)$ & $0.062(8)$ \\
\hline
\end{tabular}




\begin{tabular}{|c|c|c|c|c|}
\hline H15 & 0.5403 & -0.0875 & 0.7945 & $0.074^{*}$ \\
\hline C9 & $0.8188(18)$ & $0.0831(18)$ & $0.9886(10)$ & $0.065(8)$ \\
\hline H9 & 0.8140 & 0.0714 & 1.0236 & $0.079^{*}$ \\
\hline C33 & $0.8165(19)$ & $0.386(2)$ & $0.9083(16)$ & $0.080(11)$ \\
\hline H33 & 0.8528 & 0.3948 & 0.9367 & $0.096^{*}$ \\
\hline C26 & $0.5562(16)$ & $0.283(2)$ & $0.7001(13)$ & $0.068(8)$ \\
\hline H26 & 0.5225 & 0.2650 & 0.6722 & $0.081^{*}$ \\
\hline C6 & $0.9511(13)$ & $0.0741(19)$ & $0.8998(15)$ & $0.074(10)$ \\
\hline H6 & 0.9882 & 0.0487 & 0.9200 & $0.089^{*}$ \\
\hline C30 & $0.707(3)$ & $0.4726(18)$ & $0.7933(17)$ & $0.098(15)$ \\
\hline H30 & 0.7226 & 0.5212 & 0.7963 & $0.118^{*}$ \\
\hline
\end{tabular}

\section{3. Materials and physical measurements}

Elemental analysis $(\mathrm{C}, \mathrm{H}$, and $\mathrm{N})$ were performed on a Perkin-Elmer $2400 \mathrm{CHN}$ Elemental Analyzer. P, Ni, V and $W$ were determined on a ICP-AES Inductively Coupled Plasma spectrometer.

Morphology and EDAX measurements were performed on Philips XL30 Scanning electronic microscope along with an integrated EDAX X-ray system.

Powder X-Ray spectra in the range of $3-40^{\circ}(2 \theta)$ was carried out using Powder X-Ray diffractometer BRUKER D8-ADVANCE with CuKa $(\lambda=1,5406 \AA)$ radiation at room temperature.

The infrared spectra were recorded with a Perkin Spectrum 1000 spectrometer with a $\mathrm{KBr}$ pellet in the $4000-$ $400 \mathrm{~cm}^{-1}$ region.

UV-Vis spectra were recorded on a Perkin Elmer Lambda 19 spectrophotometer in the $200-800 \mathrm{~nm}$ range.

Emission spectra were obtained on a Perkin-Elmer LS55 spectrofluorometer equipped with a $450 \mathrm{~W}$ xenon lamp as the excitation source using solid samples at room temperature.

\section{RESULTS AND DISCUSSION}

\section{1. Crystal structure}

The title Compound was prepared by solvothermal reaction conditions in good yields as dark green blocks. Its morphology is detected by scanning electron microscopy SEM and the qualitative analysis of these crystals by electron microscope probe revealed the presence of the $\mathrm{W}, \mathrm{V}, \mathrm{Ni}, \mathrm{O}, \mathrm{N}$, and $\mathrm{C}$ atoms (Figure 1) as well as confirmed by elemental analysis.
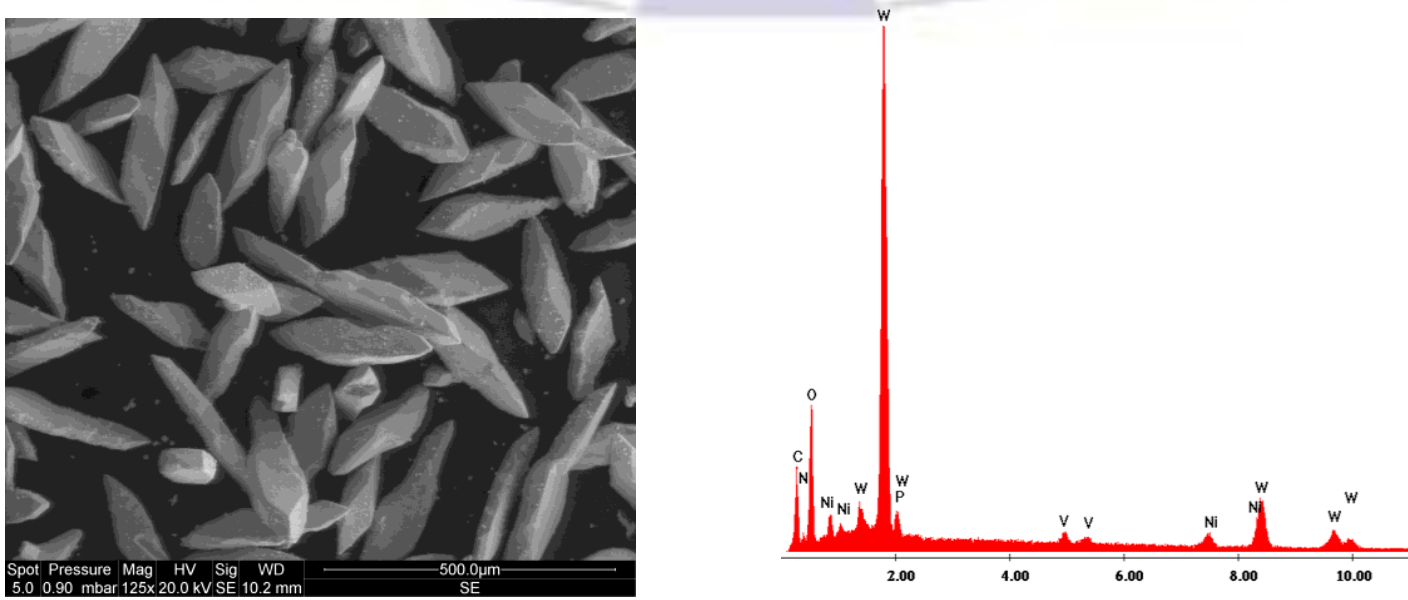

Figure 1. The SEM micrograph and EDAX pattern of $\left[\mathrm{Ni}_{(}\left(\mathrm{C}_{12} \mathrm{H}_{8} \mathrm{~N}_{2}\right)_{3}\right]_{2}\left[\mathrm{PVW}_{11} \mathrm{O}_{40}\right] .4 \mathrm{H}_{2} \mathrm{O}$ 
The XRD pattern depicted in Figure 2, shows the purity of the as synthesized sample. Moreover the presence of the typical reflexions at $8^{\circ}<2 \theta<10^{\circ}$ well prove that the sample possesses well-defined Keggin structure [29-31].

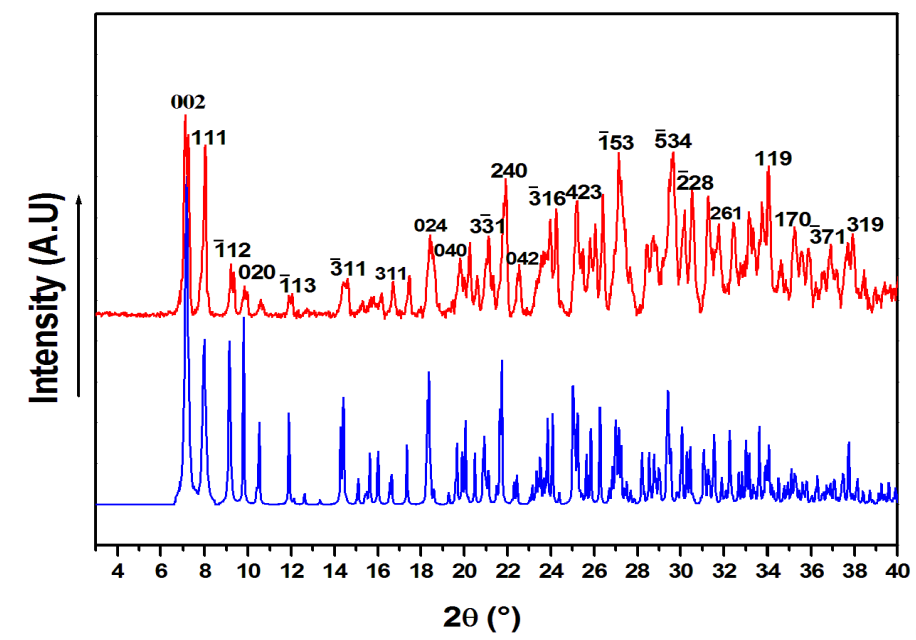

Figure 2. Experimental (red) and theoretical (blue) powder X-ray diffraction patterns of $\left[\mathrm{Ni}\left(\mathrm{C}_{12} \mathrm{H}_{8} \mathrm{~N}_{2}\right)_{3}\right]_{2}\left[\mathrm{PVW}_{11} \mathrm{O}_{40}\right] \cdot 4 \mathrm{H}_{2} \mathrm{O}$

Crystal structure analysis reveals that the structure of $\left[\mathrm{Ni}\left(\mathrm{C}_{12} \mathrm{H}_{8} \mathrm{~N}_{2}\right)_{3}\right]_{2}\left[\mathrm{PVW}{ }_{11} \mathrm{O}_{40}\right] \cdot 4 \mathrm{H}_{2} \mathrm{O}$ is derived from $\alpha$-Keggin-type polyanion $\left[\mathrm{PX}_{12} \mathrm{O}_{40}\right]^{n-}$ with asymmetric unit made up of one $\mathrm{Ni}^{2+}$ ion, three phenanthroline ligands, a half $\alpha$-Keggin-type polyanion and two uncoordinated water molecules. As central phosphorus atom is located on inversion centre site, the whole formula unit is generated by this element symmetry (Figure 3 ). The elemental analysis and EDAX patterns supported by IR spectra (Figure 6) measurements as well as charge balance calculations, indicate that there is a monosubstituted vanadium Keggin-type $\left[\mathrm{PVW}_{11} \mathrm{O}_{40}\right]$ cluster. The assignment of oxidation states for the tungsten and vanadium atoms is confirmed by bond valence sum calculations using an empirical formula of bond valence, $S=\exp [-(R$ $\left.-R_{0}\right) / 0.37$ ] ( $S=$ bond valence, $R$ bond length) [32]. The BVS results show that vanadium atom has $+\mathrm{V}$ oxidation state (average 5.0485 valence units for the $\mathrm{V}$ atom), while tungsten atoms have $+\mathrm{VI}$ oxidation state (average 6.3309 valence units for the $\mathrm{W}$ atoms). These oxidation states are identical with the charge balance considerations and so consistent with the expected $\left[\mathrm{PV}^{+\mathrm{V}} \mathrm{W}^{+\mathrm{VI}_{11}} \mathrm{O}_{40}\right]^{4-}$ subunits (abbreviated to PVW11). However, the unique $\mathrm{V}$ atom and $11 \mathrm{~W}$ atoms are in a site occupancy disorder state over the whole $\alpha$-Keggin polyanion, $\left[\mathrm{PX}_{12} \mathrm{O}_{40}\right]^{n-}$. Attempts to definitely locate the $\mathrm{V}$ atom were unsuccessful due to its low occupancy. As a result, each $\mathrm{X}$ site in the polyanion structure was assigned as $11 / 12 \mathrm{~W}$ and $1 / 12 \mathrm{~V}$ while the $\mathrm{V}$ was constrained in the final refinement. This statistically disorder is very common for this kind of heteropolyoxotungstate as regarded in bibliography [23-25].

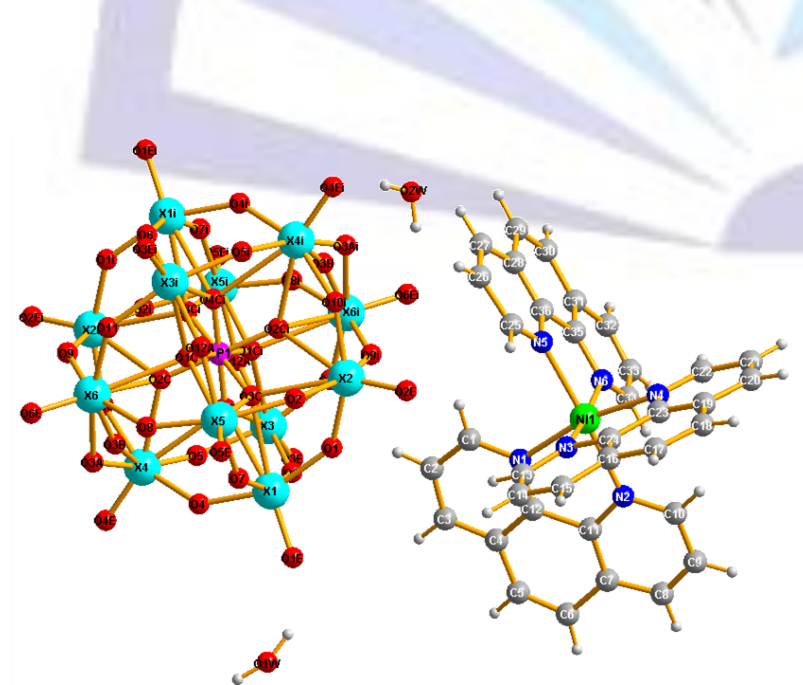

(a)

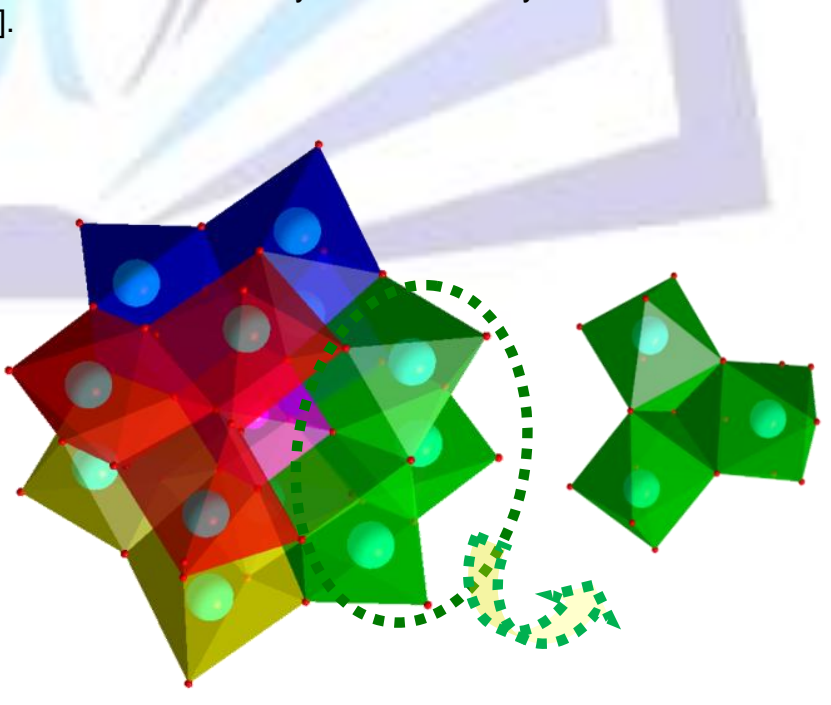

(b)

Figure 3. (a) The molecular structure of $\left[\mathrm{Ni}\left(\mathrm{C}_{12} \mathrm{H}_{8} \mathrm{~N}_{2}\right)_{3}\right]_{2}\left[\mathrm{PVW}_{11} \mathrm{O}_{40}\right] \cdot 4 \mathrm{H}_{2} \mathrm{O}$ [Symmetry code: (i) 1-x, 1-y, 1-z]; (b) Polyhedral views of the PV1W11 polyoxoanion and the trimetallic $\left\{\mathrm{X}_{3} \mathrm{O}_{13}\right\}$ subunits with $\mathrm{X}=\mathrm{W} / \mathrm{V}$ 
The PVW11 polyanion is formed from twelve $\mathrm{XO}_{6}$ octahedra enwrapping a $\mathrm{PO}_{8}$ cube (Figure 3) with oxygen atom site occupancy of 0.5 . The central phosphorus atom shares its oxygen atoms with four trimetallic $\mathrm{X}_{3} \mathrm{O}_{13}$ groups, each of which is made up of three edge-sharing $\left\{\mathrm{XO}_{6}\right\}$ octahedra, and the $\left\{\mathrm{X}_{3} \mathrm{O}_{13}\right\}$ subunits are joined to each other by corner-sharing mode. The $\mathrm{P}-\mathrm{O}$ bond distances range $1.46(2)-1.58(2) \AA$ and $\mathrm{O}-\mathrm{P}-\mathrm{O}$ bond angles interval $105.2(13)-112.9(14)^{\circ}$. Commonly, the $\mathrm{X}-\mathrm{O}$ bond distances are grouped into three sets: $\mathrm{X}-\mathrm{Ot}, \mathrm{X}-\mathrm{Ob}$ and $\mathrm{X}-\mathrm{Oc}$ (with Ot: terminal oxygen atoms of type $\mathrm{X}-\mathrm{Ot}, \mathrm{Ob}$ : bridging oxygen atoms of type $\mathrm{X}-\mathrm{Ob}-\mathrm{X}$ and Oc: central oxygen atoms of type $\mathrm{X}-\mathrm{Oc}-\mathrm{P}$ ) which are respectively ranged between 1.648 (15)-1.693 (17), 1.83 (2)- 2.08 (3) $\AA$ and 2.37 (2) -2.51 (2) $\AA$ as shown in Table 3. The $\mathrm{X}-\mathrm{O}-\mathrm{X}$ bond angles are in the range from $91.1(9)^{\circ}$ to $142.2(14)^{\circ}$ (Table 3). These geometrical features are comparable to those observed in related structure of substituted keggin type clusters $[23-25,33] . \mathrm{The}^{2+} \mathrm{Ni}^{2+}$ ion metal is also coordinated by six nitrogen atoms from three chelating 1,10-phenanthroline ligands to form a rather regular $\mathrm{MN}_{6}$ octahedron with bond lenghts around $\mathrm{Ni}$ are $2.11(2)-2.16(2) \AA(\mathrm{Ni}-\mathrm{N}), 77.3(7)-172.8(8)^{\circ}(\mathrm{N}-\mathrm{Ni}-\mathrm{N})(\mathrm{Table} 3)$. The distortion index [34] of $\mathrm{Ni}^{2+}$ octahedron is 0.023 . By comparison with the Co-complex in $\left[\mathrm{Co}(\text { phen })_{3}\right]_{2}\left[\mathrm{PVW}_{11} \mathrm{O}_{40}\right] .2 \mathrm{H}_{2} \mathrm{O}$ [25], we can observe that the distortion decreases to 0.015 for $\mathrm{Co}^{2+}$ octahedron which may be in relation with the ionic radius of metal cations.

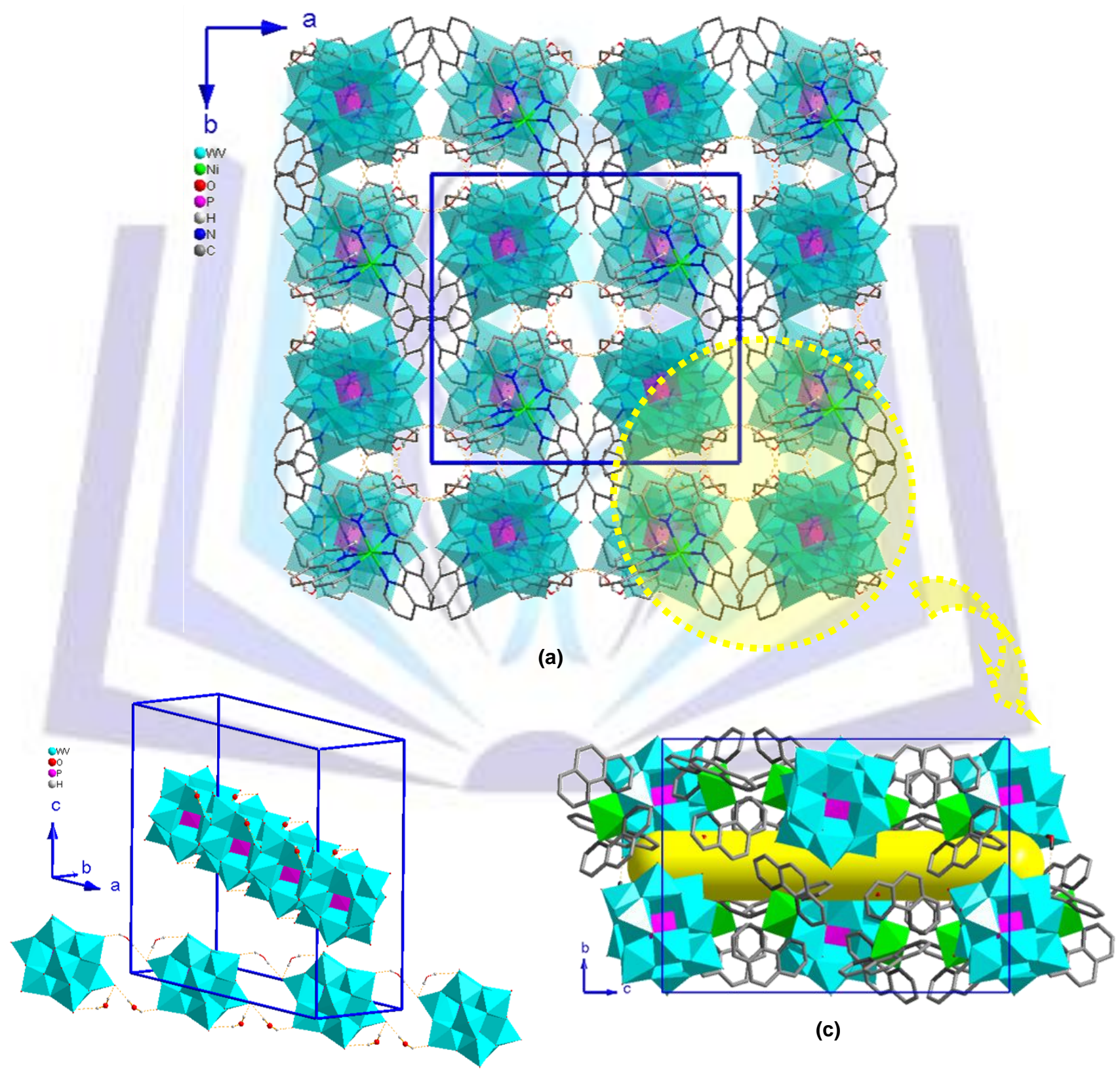

(b)

Figure 4. (a) Packing diagram of the structure viewed along c-axis (b) perspective view of the $\left[\mathrm{PV}_{1} \mathrm{~W}_{11} \mathrm{O}_{40}\left(\mathrm{H}_{2} \mathrm{O}\right)_{4}\right]_{n}{ }^{4-}$ ribbons along [110] and [110] crystallographic directions (c) 3D-supramolecular architecture in the structure featuring the channels voids along c-axis. 
Table 3. Selected bond lengths $(\AA)$, and angles $\left(^{\circ}\right)$ in $\left[\mathrm{Ni}\left(\mathrm{C}_{12} \mathrm{H}_{8} \mathrm{~N}_{2}\right)_{3}\right]_{2}\left[\mathrm{PVW}_{11} \mathrm{O}_{40}\right] \cdot 2 \mathrm{H}_{2} \mathrm{O}$

\begin{tabular}{|c|c|c|c|}
\hline & bond lenaths $(\AA)$ & bond anales $\left({ }^{\circ}\right)$ & \\
\hline \multicolumn{4}{|c|}{$\mathrm{WiO}_{6}$ octahedra } \\
\hline W1-O1E & $1.693(17)$ & $\mathrm{W} 2-\mathrm{O} 1-\mathrm{W} 1$ & $139.8(13)$ \\
\hline W1-O4 & $1.874(15)$ & $\mathrm{W} 5-\mathrm{O} 2-\mathrm{W} 2$ & $135.5(13)$ \\
\hline W1-O1 & $1.897(16)$ & $\mathrm{W} 4-\mathrm{O} 3 \mathrm{~A}-\mathrm{W} 6$ & $123.0(15)$ \\
\hline W1-O7 & 1.899 (19) & $\mathrm{W} 1-\mathrm{O} 4-\mathrm{W} 4$ & $139.6(10)$ \\
\hline W1-O6 & $1.903(17)$ & $\mathrm{W} 3-\mathrm{O} 5-\mathrm{W} 4$ & $139.0(10)$ \\
\hline W2-O2E & $1.657(15)$ & $\mathrm{W} 3-\mathrm{O} 6-\mathrm{W} 1$ & $139.1(11)$ \\
\hline W2-O10 & $1.88(2)$ & $\mathrm{W} 5-\mathrm{O} 7-\mathrm{W} 1$ & $140.6(14)$ \\
\hline W2-O1 & $1.883(19)$ & $\mathrm{W} 5-\mathrm{O} 8-\mathrm{W} 6$ & $142.2(14)$ \\
\hline W2-09 & $1.90(2)$ & $\mathrm{W} 6-\mathrm{O} 9-\mathrm{W} 2$ & $140.0(12)$ \\
\hline W2-O2 & $1.938(19)$ & $\mathrm{W} 2-\mathrm{O} 10-\mathrm{W} 4^{\mathrm{i}}$ & $139.0(13)$ \\
\hline W2-O2C & $2.43(3)$ & $\mathrm{W} 6{ }^{\mathrm{i}}-\mathrm{O} 11-\mathrm{W} 3$ & $139.1(15)$ \\
\hline W3-O3E & $1.666(16)$ & $\mathrm{W} 5-\mathrm{O} 12 \mathrm{~A}-\mathrm{W} 3^{\mathrm{i}}$ & $138.6(18)$ \\
\hline W3-O6 & $1.878(17)$ & $\mathrm{W} 2-\mathrm{O} 1-\mathrm{W} 1$ & $139.8(13)$ \\
\hline W3-O5 & $1.883(15)$ & $\mathrm{W} 5-\mathrm{O} 2-\mathrm{W} 2$ & $135.5(13)$ \\
\hline W3-O12A' & $1.90(2)$ & $\mathrm{W} 4-\mathrm{O} 3 \mathrm{~A}-\mathrm{W} 6$ & $123.0(15)$ \\
\hline W3-O11 & $1.909(18)$ & $\mathrm{W} 1-\mathrm{O} 4-\mathrm{W} 4$ & $139.6(10)$ \\
\hline W3-O1C & 2.39 (3) & $\mathrm{W} 3-\mathrm{O} 5-\mathrm{W} 4$ & $139.0(10)$ \\
\hline W4-O4E & $1.677(17)$ & $\mathrm{W} 3-\mathrm{O} 6-\mathrm{W} 1$ & $139.1(11)$ \\
\hline W4-O4 & $1.899(15)$ & $\mathrm{W} 5-\mathrm{O} 7-\mathrm{W} 1$ & $140.6(14)$ \\
\hline W4-O10' & 1.907 (17) & $\mathrm{W} 5-\mathrm{O} 8-\mathrm{W} 6$ & $142.2(14)$ \\
\hline W4-O5 & $1.923(15)$ & $\mathrm{W} 6-\mathrm{O} 9-\mathrm{W} 2$ & $140.0(12)$ \\
\hline W4-O3A & $1.94(3)$ & $\mathrm{W} 2-\mathrm{O} 10-\mathrm{W} 4^{\mathrm{i}}$ & $139.0(13)$ \\
\hline W4-O2C' & $2.37(2)$ & $\mathrm{W} 6-\mathrm{O} 11-\mathrm{W} 3$ & $139.1(15)$ \\
\hline W5-O5E & $1.648(15)$ & $\mathrm{W} 5-\mathrm{O} 12 \mathrm{~A}-\mathrm{W} 3^{\mathrm{i}}$ & $138.6(18)$ \\
\hline W5-O8 & $1.83(2)$ & $\mathrm{W} 2-\mathrm{O} 1-\mathrm{W} 1$ & $139.8(13)$ \\
\hline W5-O7 & $1.87(2)$ & $\mathrm{W} 5-\mathrm{O} 2-\mathrm{W} 2$ & $135.5(13)$ \\
\hline W5-O12A & $1.88(2)$ & $\mathrm{W} 4-\mathrm{O} 3 \mathrm{~A}-\mathrm{W} 6$ & $123.0(15)$ \\
\hline W5-O2 & $1.90(2)$ & $\mathrm{W} 1-\mathrm{O} 4-\mathrm{W} 4$ & $139.6(10)$ \\
\hline W5-O1C' & $2.46(3)$ & $\mathrm{W} 3-\mathrm{O} 5-\mathrm{W} 4$ & $139.0(10)$ \\
\hline W6-O6E & $1.665(16)$ & $\mathrm{W} 3-\mathrm{O} 6-\mathrm{W} 1$ & $139.1(11)$ \\
\hline W6-O9' & $1.87(2)$ & $\mathrm{W} 5-\mathrm{O} 7-\mathrm{W} 1$ & $140.6(14)$ \\
\hline W6-O11' & $1.90(2)$ & W5-O8-W6 & $142.2(14)$ \\
\hline W6-O8 & $1.90(2)$ & $\mathrm{W} 6-\mathrm{O} 9-\mathrm{W} 2$ & $140.0(12)$ \\
\hline W6-O3A & $2.08(3)$ & W2-O10-W4i & 139.0 (13) \\
\hline
\end{tabular}




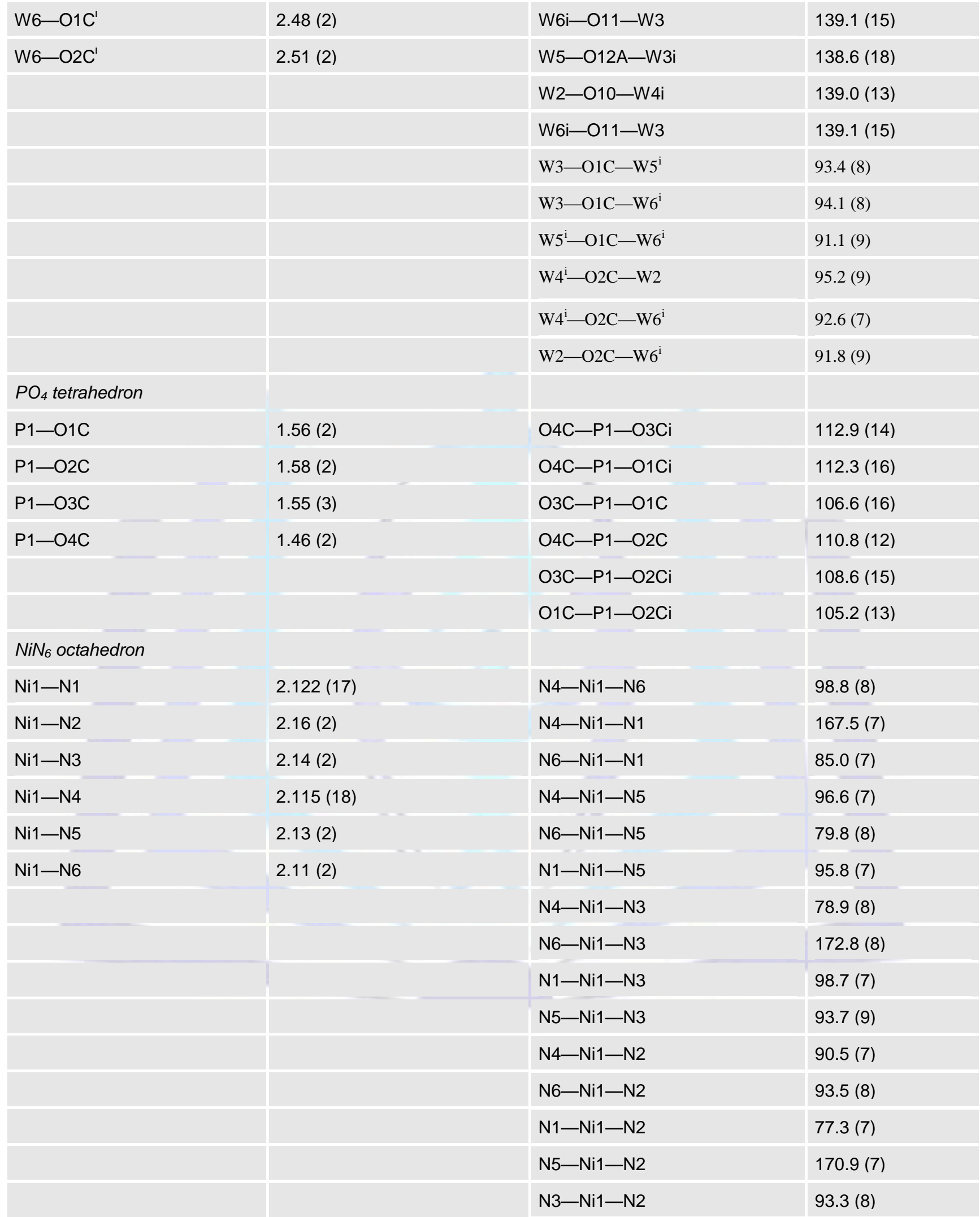

Symmetry code: (i) $-x+3 / 2,-y+1 / 2,-z+1$.

The crystal packing of the structure shows that the discrete PVW11 subunits are interconnected through water molecules via $\mathrm{O}-\mathrm{H}$... O hydrogen bonding interactions with O...O separation ranging from 2.65 (3) to 3.11 (3) $\AA$ (Table 4), to perform alternating $\left[\mathrm{PV}_{1} \mathrm{~W}_{11} \mathrm{O}_{40}\left(\mathrm{H}_{2} \mathrm{O}\right)_{4}\right]_{\mathrm{n}}^{4-}$ ribbons extending along [110] and [1 $\left.\overline{1} 0\right]$ crystallographic directions respectively at $\mathrm{z}=0$ and $z=1 / 2$ (Figure $4 b$ ). The so-obtained 1D-subnetworks stack together by the metal-organic moieties thanks to weak $C-$ 
H...O ( mean C...O = $3.22 \AA$ ) (Table 4) (Figure 5) and electrostatic interactions so as to build 3D-supramolecular networks generating vacant $1 \mathrm{D}$-channels along c-axis as can be seen in Figure 4.

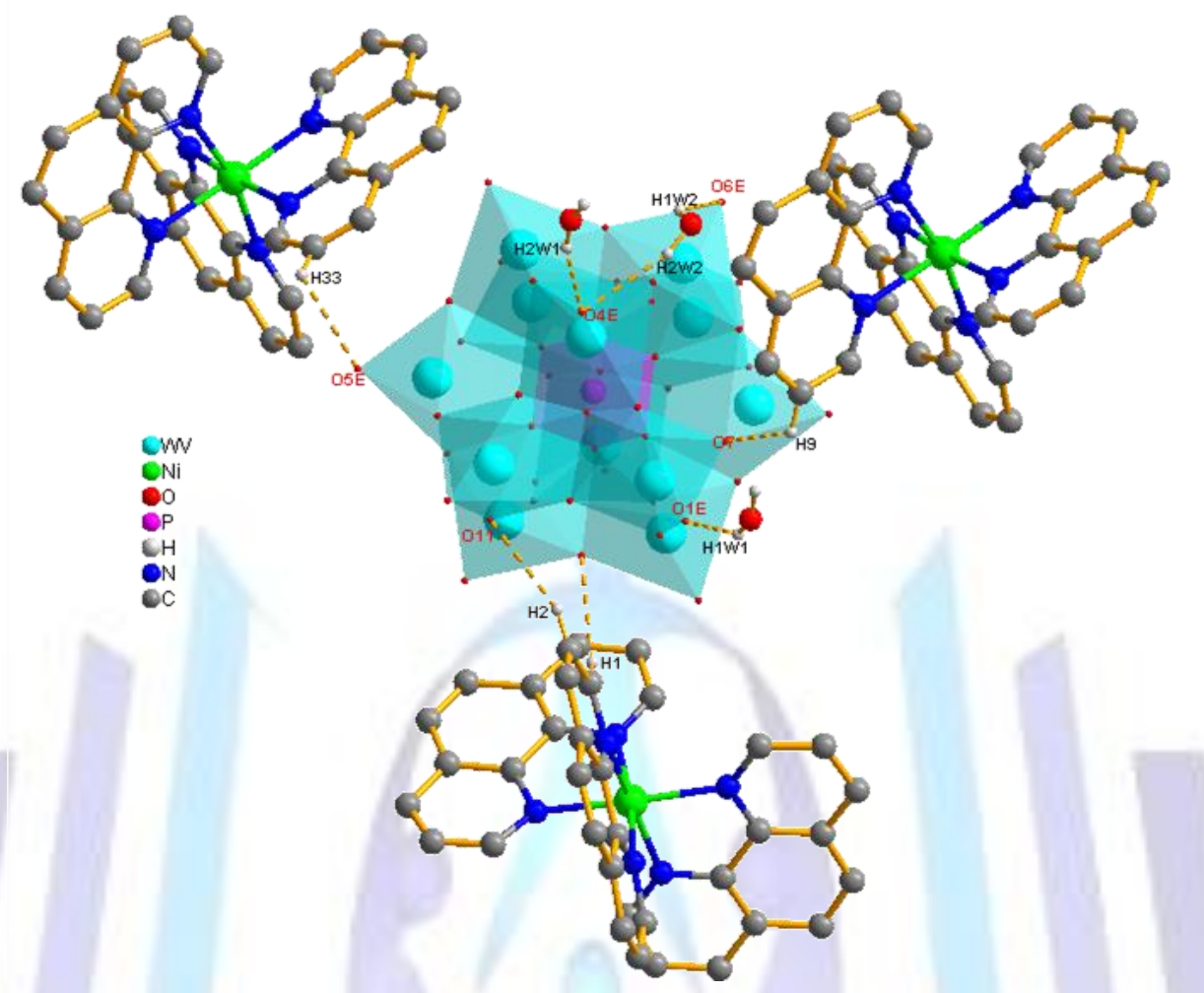

Figure 5. View of $\mathrm{O}-\mathrm{H} . . . \mathrm{O}$ and $\mathrm{C}-\mathrm{H}$...O intermolecular interactions in $\left[\mathrm{Ni}\left(\mathrm{C}_{12} \mathrm{H}_{8} \mathrm{~N}_{2}\right)_{3}\right]_{2}\left[\mathrm{PVW}_{11} \mathrm{O} \mathrm{O}_{40}\right] .4 \mathrm{H}_{2} \mathrm{O}$. $\mathrm{Hydrogen}$ atoms of phenanthroline ligands not included in $\mathrm{H}$-bond scheme are omitted for clarity.

Table 4. Hydrogen-bond geometry $\left(\AA,^{\circ}\right)$ in $\left[\mathrm{Ni}\left(\mathrm{C}_{12} \mathrm{H}_{8} \mathrm{~N}_{2}\right)_{3}\right]_{2}\left[\mathrm{PVW}_{11} \mathrm{O}_{40}\right] \cdot 4 \mathrm{H}_{2} \mathrm{O}$

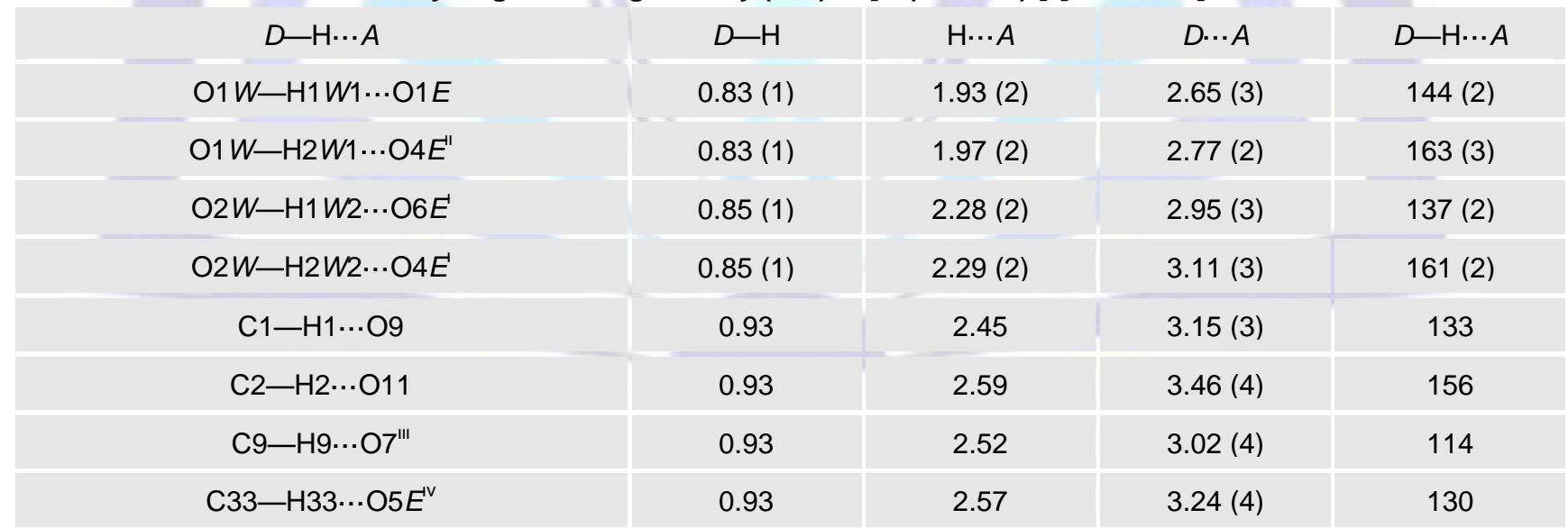

Symmetry codes: (i) $-x+3 / 2,-y+1 / 2,-z+1$; (ii) $-x+2,-y,-z+1$; (iii) $x,-y, z+1 / 2$; (iv) $-x+3 / 2, y+1 / 2,-z+3 / 2$.

\section{2. IR investigation}

The IR spectrum (Figure 6) of the reported sample (Figure 6) exhibits the Keggin characteristic peaks at 966 , $885,791 \mathrm{~cm}^{-1}$ attributed respectively to $v\left(\mathrm{X}=\mathrm{O}_{\mathrm{d}}\right), v\left(\mathrm{X}-\mathrm{O}_{\mathrm{b}}-\mathrm{X}\right)$ and $v\left(\mathrm{X}-\mathrm{O}_{\mathrm{c}}-\mathrm{X}\right)$ with $\mathrm{X}=\mathrm{W} / \mathrm{V}$, while the $v(\mathrm{P}-\mathrm{O})$ splits into two bands $1099,1072 \mathrm{~cm}^{-1}$ because of the lower symmetry so as well confirm the presence of monosubstituted vanadium keggin-type clusters [35-36]. The bands between 1700 and $1100 \mathrm{~cm}^{-1}$ are characteristic absorption of 1,10-phen ligand [37-39]. The broad band around $3450 \mathrm{~cm}^{-1}$ could be due to absorption of water of crystallization. 


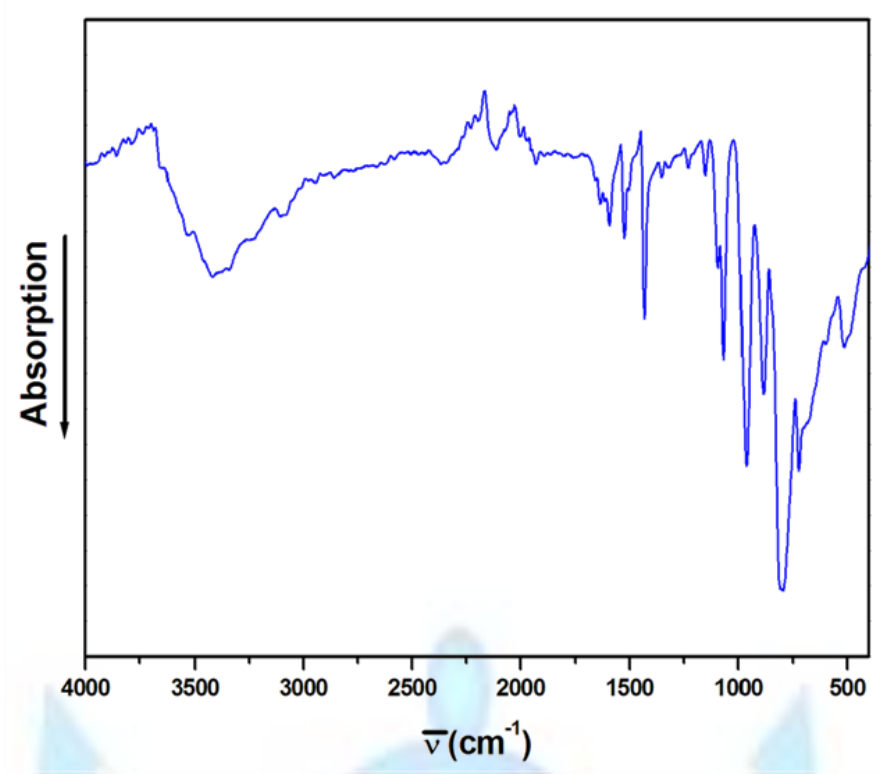

Figure 6. IR spectrum of $\left[\mathrm{Ni}\left(\mathrm{C}_{12} \mathrm{H}_{8} \mathrm{~N}_{2}\right)_{3}\right]_{2}\left[\mathrm{PVW}_{11} \mathrm{O}_{40}\right] .4 \mathrm{H}_{2} \mathrm{O}$

\section{3. UV/Vis properties and optical band gaps}

The UV-Vis electronic spectra of $\left[\mathrm{Ni}\left(\mathrm{C}_{12} \mathrm{H}_{8} \mathrm{~N}_{2}\right)_{3}\right]_{2}\left[\mathrm{PVW}_{11} \mathrm{O}_{40}\right] \cdot 4 \mathrm{H}_{2} \mathrm{O}$ and pure phenanthroline in DMSO solution at room temperature are depicted in Figure 7A. The UV-Vis electronic spectrum of the complex exhibits broad and strong bands spreading between $200 \mathrm{~nm}$ and $350 \mathrm{~nm}$. The strong band with a maximum centered at $295 \mathrm{~nm}$ is relatively close to this observed for the free phen ligand $\left(\lambda_{\max }=300 \mathrm{~nm}\right)$ and corresponds to intraligand and ligand to-metal charge transfer (LMCT) of Ni metal [40-41]. In addition, the broad band centered at about $230 \mathrm{~nm}$ may be due to $\mathrm{O}_{\mathrm{t}} \rightarrow \mathrm{X}$ and $\mathrm{O}_{\mathrm{b} / \mathrm{c}} \rightarrow \mathrm{X}(\mathrm{X}=$ $\mathrm{W}$ and $\mathrm{V}$ ) charge transfer (LMCT), characteristic of Keggin anions [42-43]. Moreover the electronic spectra of the compound provided by using the Tauc model [44], optical band gaps of ca. $3.9 \mathrm{eV}$ as reported in Figure 7B, suggesting that the materials may possess semiconducting properties [45].

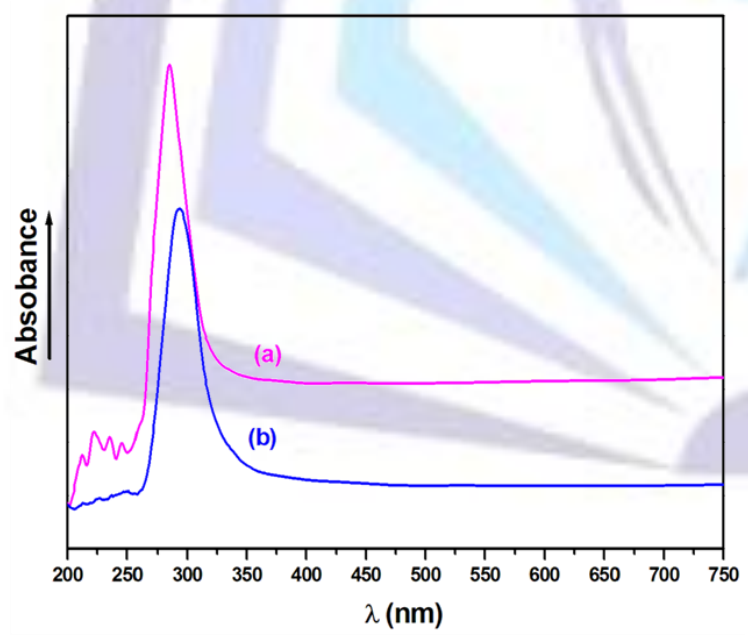

A

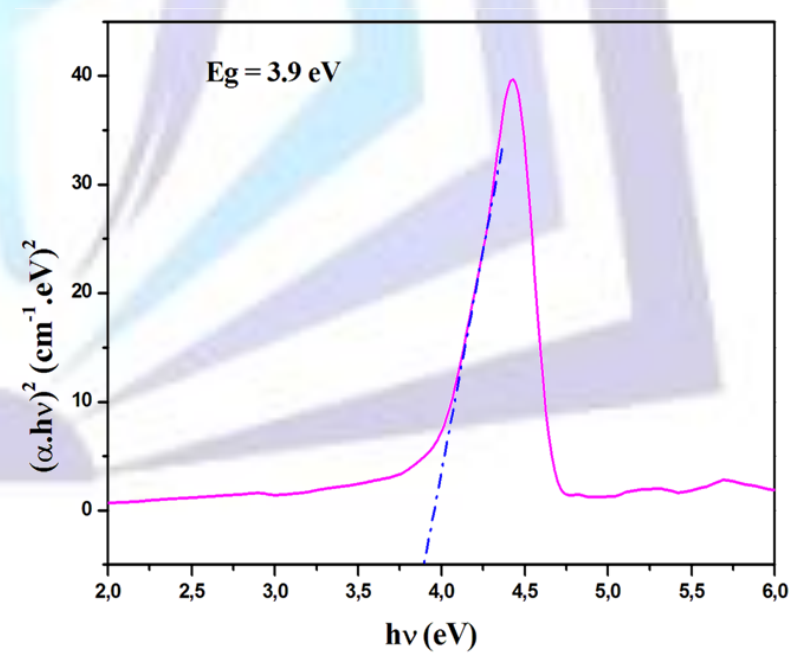

B

Figure 7. (A) UV absorption spectra obtained from DMSO solutions of $2 \times 10^{-4} \mathrm{~mol}^{-1} \mathrm{~L}^{-1}$ of (a) $\left[\mathrm{Ni}\left(\mathrm{C}_{12} \mathrm{H}_{8} \mathrm{~N}_{2}\right)_{3}\right]_{2}\left[\mathrm{PVW}_{11} \mathrm{O}_{40}\right] \cdot 4 \mathrm{H}_{2} \mathrm{O}$ and (b) free phenanthroline. (B). Band gap determination for the complex according the Tauc model. 


\section{4. Fluorescence properties}

The photoluminescent properties of free phen and the compound in the solid state at room temperature have been investigated. The emission spectrum of the compound (Figure $8(\mathrm{~b})$ ) shows broad emission bands red-shifted in comparison to the free phen ligand (Figure 8(a)) with maxima at $422 \mathrm{~nm}, 481 \mathrm{~nm}, 530 \mathrm{~nm}$ upon excitation at ca. $295 \mathrm{~nm}$. The reported material exhibits blue photoluminescence which can be assigned to the emission to intraligand and ligand tometal charge transfer (LMCT) [46-49] as evidenced by UV-visible analysis.

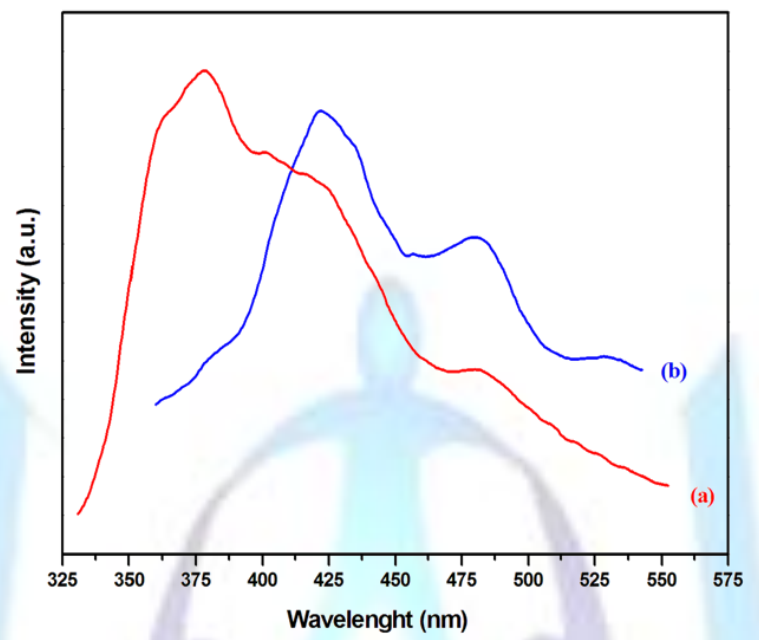

Figure 8. Solid-state fluorescence emission spectra at room temperature of (a) free phen and (b) $\left[\mathrm{Ni}\left(\mathrm{C}_{12} \mathrm{H}_{8} \mathrm{~N}_{2}\right)_{3}\right]_{2}\left[\mathrm{PVW}_{11} \mathrm{O}_{40}\right] .4 \mathrm{H}_{2} \mathrm{O}(\lambda$ excitation at $295 \mathrm{~nm})$.

\section{Conclusion}

In this paper, we have reported and characterized a new hybrid organic-inorganic mono-V-substituted Keggintype tungstophosphate, $\left[\mathrm{Ni}\left(\mathrm{C}_{12} \mathrm{H}_{8} \mathrm{~N}_{2}\right)_{3}\right]_{2}\left[\mathrm{PVW}_{11} \mathrm{O}_{40}\right] .4 \mathrm{H}_{2} \mathrm{O}$. XRD analysis shows that is characterized by $1 \mathrm{D}$ alternating $\left[\mathrm{PV}_{1} \mathrm{~W}_{11} \mathrm{O}_{40}\left(\mathrm{H}_{2} \mathrm{O}\right)_{4}\right]_{\mathrm{n}}^{4-}$ subnetworks extending along [110] and [110] at $\mathrm{z}=0$ and $\mathrm{z}=1 / 2$. These ribbons are stacked together by means $\left[\mathrm{Ni}(\text { phen })_{3}\right]^{2+}$ subunits thanks to $\mathrm{C}-\mathrm{H}$...O and electrostatic interactions so as to build 3D-hydrogen bondsupported supramolecular networks generating vacant 1D-channels along c-axis. Meanwhile, the electronic investigations provide on the one hand, the optic band gaps describing the semiconductor behavior owing to the electron delocalization and on the other hand, the fluorescent property of the reported material.

\section{ACKNOWLEDGMENTS}

The authors express their appreciation to the CNRS 6230 UFR sciences and techniques of Nantes in France for supporting the work.

\section{REFERENCES}

[1] M. T. Pope and A. Müller, Polyoxometalate chemistry: an old field with new dimensions in several disciplines, Angew. Chem. Int. Ed. Engl., 1991, 30, 34-48.

[2] P. Gouzerh and A. Proust, Chem. Rev., 1998, 98, 77-112.

[3] V. Soghomonian, Q. Chen, R. C. Haushalter, J. Zubieta, C. O'Connor, Science 1993, 259, 1596-1599.

[4] J. T. Rhule, C. L. Hill, D. A. Judd and R. F. Schinazi, Chem. Rev. 1998, 98, 327-357.

[5] G. Centi, Catal. Today 1993, 16, 5-26.

[6] X. M. Zhang, M. L. Tong, X. M. Chen, Chem. Commun. 2000, 1817-1818.

[7] L. H. Bi, R. D. Huang, J. Peng, E. B. Wang, Y. B. Wang, C. W. Hu, Dalton Trans. 2001, 121-129.

[8] M. I. Khan , R. C. Haushalter , C. J. O'Connor , C. Tao and J. Zubieta, Chem. Mater. 1995, 7(4), 593-595.

[9] S. Ikeda, S. Nishiya, A. Yamamoto, T. Yamase, C. Nishimura, E. De Clercq, J. Med. Virology 1993, 41(3), $191-195$.

[10] A. Flütsch, T. Schroeder, M. G. Grütter, G. R. Patzke, Bioorg. Med. Chem. Lett. 2011, 21, 1162-1166. 
[11] T. Yamase, J. Mater. Chem., 2005, 15, 4773-4782.

[12] N. Essayem, Y. Ben Taarit, C. Feche, P. Y. Gayraud, G. Sapaly, C. Naccache, J. Catal. 2003, 219, 97-106.

[13] W. Kuang, A. Rives, M. Fournier, R. Hubaut, Catal. Lett 2002, 79(1), 133-135.

[14] P. Sami, T. D. Anand, M. Premanathan, K. Rajasekaran, Transition Metal Chem. 2012, 35(8), 1019-1025.

[15] P. Sami, K. Venkateshwari, N. Mariselvi, A. Sarathi, K. Rajasekaran, Transition Metal Chem. 2012, 34(7), 733-737.

[16] R. Murugesan, P. Sami, T. Jeyabalan, A. Shunmugasundaram, Transition Metal Chem. 1998, 23(5), 583-588.

[17] X. Xue, F. Song, B. Ma, Y. Yu, C. Li, Y. Ding, Catal. Commu. 2013, 33, 61-65.

[18] T. Yamaura, K. Kamata, K. Yamaguchi, N. Mizuno, Catal. Today 2013, 203, 76-80.

[19] D. R. Park, S. H. Song, U. G. Hong, J. G. Seo, J. C. Jung, I. K. Song, Catal. Lett. 2009, 132, 363-369.

[20] L. A. Glinskaya, É. N. Yurchenko, R. F. Klevtsova, L. V. Derkach, A. M. Rios, T. P. Lazarenko, J. Struct. Chem. 1989, 30(3), 427-432.

[21] R. F. Klevtsova, L. A. Glinskaya, É. N. Yurchenko, T. D. Gutsul, J. Struct. Chem. 1991, 32(5), 687-692.

[22] R. F. Klevtsova, L. A. Glinskaya, E. N. Yurchenko, L. V. Derkach, A. M. Rios, T. P. Lazarenko, J. Struc. Chem. 1990, 31(2), 285-291.

[23] C. Li, R. Cao, K. P. O'Halloran, H. Ma, L. Wu, Electrochimica Acta 2008, 54, 484-489.

[24] E. Radkov and R. H. Beer, Polyhedron 1995, 14, 2139-2143.

[25] A. Hajsalem, S. Aoun, A. Planchat, M. Rzaigui and S. Akriche Toumi, Acta Cryst. 2014, E70, m125-m126.

[26] G. M. Sheldrick, SHLXS97 and SHELXL-97, Programs for the Resolution and Refinement of Crystal Structures, University of Göttingen, Göttingen (Germany) 1997.

[27] L. J. Farrugia, University of Glasgow, Glasgow, Scotland (U. K. ) 1999.

[28] K. Brandenburg \& H. Putz, DIAMOND Crystal Impact GbR, Bonn, Germany 2005.

[29] WANG Dunjia, FANG Zhengdong, WEI Xianhong, Journal of Wuhan University of Technology-Mater. Sci. Ed. 2008, 23(2), 198-203.

[30] Z. Fumin, G. Maiping, G. Hanqing, W. Jun, Front. Chem. Eng. China 2007, 1(3), 296-299.

[31] G. L. Wang, X. J. Mi, D. M. Li, J. Natural Sciences Journal of Harbin Normal University 1997, 13, 75-79.

[32] I. D. Brown, M. O'Keefe, A. Navrotsky, Eds. Structure and Bonding in Crystals, vol. 2 Academic Press, New York, 1981, 1-30.

[33] K. Y. Lee and M. Misono, "Heteropoly compounds" in Handbook of Heterogeneous Catalysis, eds; G. Ertl, H. Knozinger, J. Weitkamp, VCH, 1997, 118-131.

[34] D. Kobashi, S. Kohara, J. Yamakawa, A. Kawahara, Acta Cryst. 1997, C53, 1523-1525.

[35] C. R. Deltcheff, M. Fournier, R. Franck and R. Thouvenot, Inorg. Chem. 1983, 22(2), 207-216.

[36] M. J. Watras, and A. V. Teplyakov, J. Phys. Chem. B 2005, 109(18), 8928-8934.

[37] D Rusu, O. Băban, I. Hauer, D. Gligor, L. David and M. Rusu, Rev. Roum. Chim. 2010, 55(11-12), 843-850.

[38] M. Yuan, Y. G. Li, E. B. Wang, C. G. Tian, L. Wang, C. W. Hu, N. H. Hu, H. Q. Jia, Inorg. Chem. 2003, 42, 36703676.

[39] Y. B. Liu, L. M. Duan, X. M. Yang, J. Q. Xu, Q. B. Zhang, Y. K. Lu, J. Liu, J. Solid State Chem. 2006, 179, $122-129$.

[40] L. E. Orgel, An Introduction to Transition-Metal Chemistry: Ligand-Field Theory; Butler \& Turner Ltd.: London, 1966.

[41] S. Arounaguiri, D. Easwaramoorthy, A Ashokkumar, A. Dattagupta and B. G Maiya, Proc. Indian Acad. Sci. (Chem. Sci.), 2000, 112(1), 1-17.

[42] T. Ueda, M. Komatsu, M. Hojo, Inorganica Chimica Acta 2003, 344, 77-84.

[43] M. T. Pope, Heteropoly and Isopoly Oxometalates, Springer-Verlag, New York, Heteropoly and Isopoly Oxometalates, Springer-Verlag, New York, 1983.

[44] J. Tauc, R. Grigorovici, A. Vancu, Phys. Status. Sol. 1966, 15, 627-637.

[45] W. Q. Kan, J. Yang, Y. Y. Liu, J. F. Ma, W. Q. Kan, J. Yang, Y. Y. Liu, J. F. Ma, Dalton Trans. 2012, 41(36), 1106211073.

[46] C. Li, R. Cao, K. P. O’Halloran, H. Ma, L. Wu, Electrochimica Acta 2008, 54, 484-489. 
[47] L. Chen, F. Jiang , Z. Lin , Y. Zhou , C. Yue , M. Hong, J. Am. Chem. Soc. 2005, 127(24), 8588-8597.

[48] K. D. Ley, K. S. Schanze, Coord. Chem. Rev. 1998, 171, 287-307.

[49] V. W. W. Yam and K. K. W. Lo, Chem. Soc. Rev. 1999, 28, 323-334. 